\title{
SEROPREVALENCIA DE Toxoplasma gondii EN LLAMAS DE UNA EMPRESA PECUARIA EN MELGAR, PUNO
}

\author{
Marco Saravia P. ${ }^{1}$, Amanda Chávez V. ${ }^{2,3}$, Eva Casas A. ${ }^{2}$, Néstor Falcón P. ${ }^{4}$ y \\ Walter Pinto S. ${ }^{5}$
}

\section{Abstract}

The objective of the present study was to determine the seroprevalence of Toxoplasma gondii in adult female llamas in two flocks of the Rural Alliance farm in the province of Melgar, Puno. A total of 157 blood samples were collected and analyzed using the indirect immunofluorescence test. The results indicated that $10.2 \pm 4.7 \%$ (16/ 157) of llamas had antibodies against $T$. gondii. One flock had $13.3 \pm 9.8 \%(6 / 45)$ seroprevalence and the other one had $8.9 \pm 5.3 \%$ (10/112); however, no significant statistical differences were found between flocks or age groups. The results showed a relatively low seroprevalence to $T$. gondii in relation to other studies in the country.

Key words: toxoplasmosis, IFI, llama, Melgar, Puno

\section{Resumen}

\begin{abstract}
El objetivo del presente trabajo fue determinar la seroprevalencia del Toxoplasma gondii en llamas hembras adultas de dos puntas de parición de la Unidad de Producción Alianza-Antacalla, en la Empresa de propiedad social "Rural Alianza", en la provincia de Melgar-Puno. Se trabajó con 157 muestras de sangre que se analizaron con la prueba de inmunofluorescencia indirecta. Se encontró que el 10.2 $\pm 4.7 \%$ (16/157) del total de llamas hembras adultas presentaron anticuerpos contra T. gondii; siendo de $13.3 \pm 9.8 \%(6 / 45)$ y $8.9 \pm 5.3 \%(10 / 112)$ en las puntas de Río Grande y Alianza, respectivamente. No se encontró diferencias estadísticas significativas entre puntas ni entre grupos etarios. Los resultados de este estudio muestran una seroprevalencia a $T$. gondii relativamente baja en relación a otros estudios similares en camélidos.
\end{abstract}

Palabras clave : toxoplasmosis, IFI, llama, Melgar, Puno

\footnotetext{
${ }^{1}$ Práctica privada

${ }^{2}$ Laboratorio de Microbiología y Parasitología Veterinaria, FMV-UNMSM

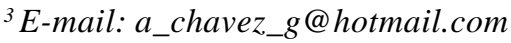

${ }^{4}$ Laboratorio de Medicina Veterinaria Preventiva, FMV-UNMSM

${ }^{5}$ Gerente General E.P.S. “Rural Alianza”. Melgar, Puno
} 


\section{INTRODUCCIÓN}

Los camélidos sudamericanos (CSA) presentan altos índices de mortalidad embrionaria (Arthur et al., 1991), así como bajos porcentajes de natalidad que fluctúan entre el 50 y 70\% (Fernández Baca, 1991); hechos que representan unos de los principales problemas en su reproducción. Hasta el momento, no se conocen con claridad a todos los factores causantes de estas deficiencias, pero es posible que la toxoplasmosis pueda afectarles y ocasionarles problemas reproductivos y mortalidad en las crías, tal como ocurre en los ovinos (Ameghino, 1991; INIA, 1990), presentando abortos y reabsorciones fetales en el primer tercio de la gestación (Hartley y Marshall, 1967).

La toxoplasmosis es causada por un protozoario intracelular, el Toxoplasma gondii, de distribución mundial, y es uno de los agentes más importantes que afectan el tracto reproductivo en las diferentes especies de animales domésticos (Arthur et al., 1991).

La mayor población de llamas está en Perú y Bolivia, donde el primero posee una población de 1'130,343 llamas, de las cuales el $37 \%$ se encuentra en Puno (MINAG, 1998). Casi la totalidad de las llamas y no menos del $90 \%$ de las alpacas pertenecen a pequeños productores (Fernández Baca, 1991), quienes mantienen rebaños de 20 a 30 animales, mayormente para autoconsumo (Franco, 1998), constituyéndose en una actividad económica de gran importancia para un vasto sector de la población altoandina (Fernández Baca, 1991).

Se han realizado pocos estudios serológicos de $T$. gondii en llamas. Gómez (2002) determinó una seroprevalencia de $28 \%$ en la Estación Experimental del INIA-PUNO y Marcas (2003) determinó una prevalencia de $48 \%$ en llamas hembras de dos fundos ganaderos de la provincia de Melgar, Puno. En otros camélidos sudamericanos se ha de- terminado la presencia de anticuerpos contra T. gondii; así, Pastor et al. (2003) halló una prevalencia de $15 \%$ en vicuñas de Puno, mediante la prueba de hemaglutinación indirecta, en tanto que Leguía et al. (1988) obtuvieron una seroprevalencia de $50 \%$ en alpacas hembras de Puno.

El presente estudio tuvo como objetivo determinar la seroprevalencia de $T$. gondii en llamas hembras adultas de una unidad de producción de la Empresa de Propiedad Social "Rural Alianza" en la provincia de Melgar, Puno.

\section{Materiales y Métodos}

\section{Animales}

El número de animales requeridos para el experimento fue de 160 llamas jóvenes y adultas, según la fórmula de proporciones en poblaciones finitas (Daniel, 1996). Sin embargo debido a condiciones climáticas desfavorables y a deficiencias en la logística, se tuvo que muestrear a 157 llamas hembras adultas (de 2 a más años de edad), de la variedad C'haku y K' ara, en febrero del 2003, dejándose de lado a los tuis (jóvenes de 1 y 2 años de edad).

Las llamas fueron de la Unidad de Producción (UP) Alianza-Antacalla de la Empresa Rural Alianza, ubicada en la provincia de Melgar, Puno, y provienen de las puntas de parición Alianza y Río Grande, las cuales se encuentran alejadas entre sí. Estos animales son criados en forma extensiva, alimentándose a base de pastos naturales.

\section{Toma de muestras}

Se tomó muestras de sangre mediante punción de la vena yugular, usando vacutainers estériles para la obtención de suero sanguíneo. Los viales de suero fueron almacenados a $-20{ }^{\circ} \mathrm{C}$ hasta su análisis en el Laboratorio de Microbiología y Parasitología 
Cuadro 1. Seroprevalencia de Toxoplasma gondii en llamas hembras adultas y su distribución según zona de parición en la U.P. Alianza-Antacalla de la E.P.S. "Rural Alianza", en la provincia de Melgar, Puno. 2003

\begin{tabular}{cccc}
\hline Variables & $\begin{array}{c}\text { Animales } \\
\text { muestreados }\end{array}$ & $\begin{array}{c}\text { Animales } \\
\text { positivos }\end{array}$ & Prevalencia \pm IC \\
\hline Zona de parición & & & \\
Alianza & 112 & 10 & $8.9 \pm 5.3$ \\
Río Grande & 45 & 6 & $13.3 \pm 9.8$ \\
Edad (años) & & & \\
$2-3$ & 44 & 4 & $9.1 \pm 8.5$ \\
$4-5$ & 26 & 4 & $15.4 \pm 13.9$ \\
$? 6$ & 87 & 8 & $9.2 \pm 6.1$ \\
\hline Total & 157 & 16 & $10.2 \pm 4.7$ \\
\hline
\end{tabular}

de la Facultad de Medicina Veterinaria de la Universidad Nacional Mayor de San Marcos, en Lima.

\section{Serología}

La detección de anticuerpos contra Toxoplasma gondii se realizó mediante la prueba de inmunofluorescencia indirecta (IFI). La muestra se consideró positiva cuando la fluorescencia amarilla verdosa se presentó en todo el contorno del taquizoíto, y negativa cuando el campo microscópico se observó de un color rojo oscuro y con ausencia total o parcial de fluorescencia intensa (verde amarillento) en todo el borde del taquizoíto.

\section{Análisis estadístico}

Los resultados obtenidos en las pruebas se expresaron en porcentajes y con intervalos de confianza del $95 \%$. Se usaron las variables de zona de parición, edad y resultado a la prueba diagnóstica. Se utilizó la prueba de regresión logística para identificar las variables que podrían ser consideradas factores de riesgo para contraer la toxoplasmosis, así como establecer su posible interrelación.

\section{Resultados}

El Cuadro 1 muestra una seroprevalencia de $10.2 \pm 4.7 \%(16 / 157)$ a $T$. gondii por inmunofluorescencia indirecta en llamas hembras adultas de la U.P. Alianza-Antacalla de la EPS Rural Alianza. No se encontró diferencias estadísticas significativas entre puntas de parición ni entre grupos etarios ( $p>0.05$ ).

Se hizo un análisis de regresión logística y de factor de riesgo con la finalidad de deteminar una posible asociación de la infección de $T$. gondii en llamas hembras adultas frente a la variable edad y punta de parición (Cuadro 2), sin resultados positivos.

\section{Discusión}

Se obtuvo una prevalencia general en llamas hembras adultas de $10.2 \pm 4.7 \%$, lo que demostraría que los animales bajo evaluación presentan una infección relativamente baja, comparado con trabajos similares en camélidos sudamericanos del país y del exterior (Cuadro 3). 
Cuadro 2. Evaluación de las variables zona de parición y edad como factores de riesgo para la infección por $T$. gondii en llamas hembras adultas de la U.P. AlianzaAntacalla de la E.P.S. "Rural Alianza", en la provincia de Melgar, Puno. 2003

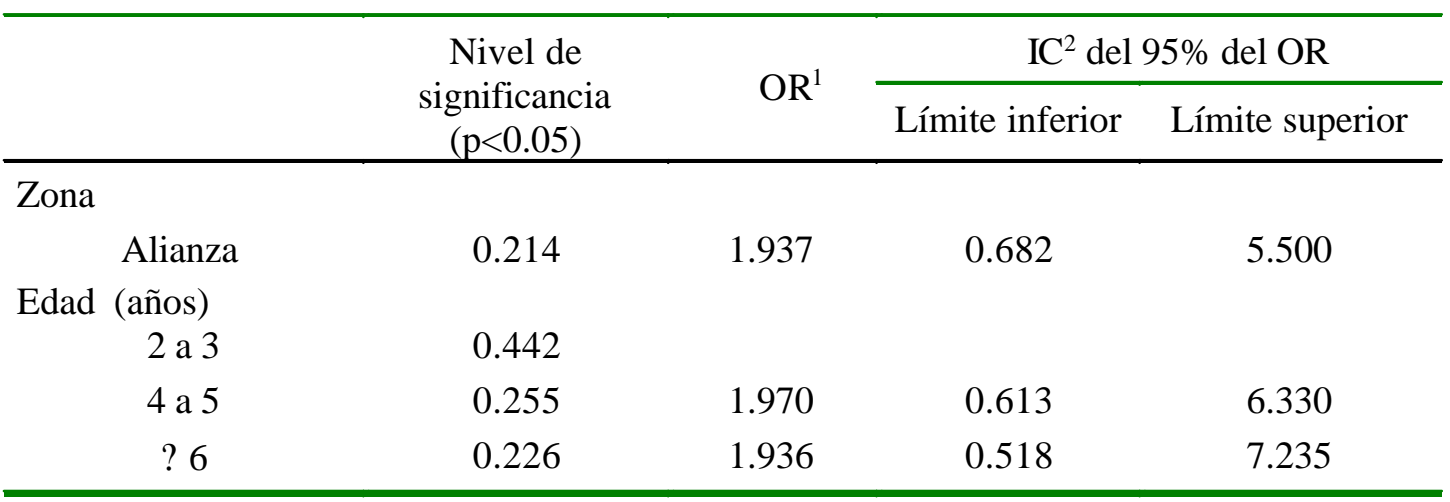

${ }_{2}^{1}$ Odds Ratio (Cuantificador de riesgo)

${ }^{2}$ Intervalo de confianza

Cuadro 3. Cuadro comparativo de reportes de seroprevalencia de Toxoplasma gondii en camélidos sudamericanos

\begin{tabular}{llccl}
\hline \multirow{2}{*}{ Especie } & Localidad & Prueba diagnóstica & $\begin{array}{c}\text { Seroprevalencia } \\
(\%)\end{array}$ & Referencia \\
\hline \multirow{3}{*}{ Llamas } & Norte de Chile & & 26 & Rojas et al., 1989 \\
& EEUU & Test de aglutinación & 34 & Dubey et al., 1992 \\
& INIA, Puno & HIA & 56 & Gómez, 2002 \\
& Melgar, Puno & IFI & 47 & Marcas, 2003 \\
\hline \multirow{6}{*}{ Alpacas } & Picotani, Puno & HAI & 50 & Leguía et al., 1988 \\
& Puno & IFI & 24 & Góngora, 1992 \\
& Altiplano de Chile & HAI & 16 & Gorman et al., 1999 \\
& INIA, Puno & HIA & 45 & Gómez, 2002 \\
& Jauja, Junín & HAI & 23 & Poma, 2003 \\
\hline Vicuñas & INIA, Puno & HIA & 15 & Pastor et al., 2003 \\
\hline
\end{tabular}

Los resultados del presente trabajo confirmarían que los animales fueron expuestos a los ooquistes de $T$. gondii en algún momento de sus vidas, y que éstos se encontrarían presentes en las pasturas de la empresa, debido probablemente a la presencia de felinos domésticos y/o silvestres en las zonas de pastoreo (Leguía et al., 1988).
Posiblemente, uno de los principales factores que haya influido en la baja prevalencia observada se debería a la menor posibilidad de contacto entre gatos domésticos y las llamas, ya que éstas son criadas en lugares alejados del centro poblado, donde se encontrarían los gatos; además, la escasa presencia de felinos silvestres, especialmente del puma, 
que podría contaminar las pasturas con ooquistes de $T$. gondii. El movimiento de las llamas en la empresa es mínimo ya que la mayor actividad ocurre con el traslado al centro poblado en la esquila, lo cual se hace cada tres a cuatro años. Una ventaja adicional estaría dada por la situación de pastoreo, donde las llamas no comparten los potreros con otras especies. Rojas (1990) menciona que los ovinos con problemas de aborto contaminan los campos de pastoreo a través de la placenta, representando un foco de infección para los camélidos sudamericanos.

Factores adicionales podrían ser las restricciones de la empresa al ingreso y tránsito de ganado, personas y vehículos ajenos a la institución. El medio ambiente frío y seco, así como la altitud de la zona (de 4,190 a 4,300 msnm) también estarían influyendo en estas bajas prevalencias. Acha y Szifres (1986) observaron diferencias de prevalencia con relación a la altitud, y Freij y Sever (1986) indican, por otro lado, que los ooquistes no sobreviven por mucho tiempo a las temperaturas de refrigeración, congelación y desecación.

Los porcentajes de prevalencias, a veces tan disímiles, que son reportados para diferentes áreas geográficas, incluso para áreas endémicas, debido en parte al carácter esporádico de la infección, hacen que los resultados no sean fácilmente extrapolables (Luzón et al., 1997), ni que se puedan realizar comparaciones válidas entre las frecuencias encontradas (D'Angelino, 1983). Además, estas diferencias pueden deberse al método de diagnóstico utilizado, pues en su gran mayoría usaron pruebas diferentes al IFI, la que tiene una mayor sensibilidad y especificidad. No obstante las ventajas de la prueba del IFI, éste tiene como desventaja la poca accesibilidad de los investigadores a microscopios de fluorescencia.

En lo que respecta a las puntas de parición, se observó una mayor prevalencia en la punta de parición de Río Grande (13.3 $\pm 9.8 \%$ ), frente a la prevalencia de la punta de Alianza $(8.9 \pm 5.3 \%)$ (Cuadro 1), donde ésta se encuentra ubicada a una altura superior. La mayor seroprevalencia de $T$. gondii en Río Grande se podría deber a la cercanía de los animales con las comunidades campesinas y pequeños productores de ganado de la zona, donde se tiene la presencia de gatos domésticos, y por lo tanto, mayores probabilidades de contaminación de los pastos con ooquistes de $T$. gondii. Sin embargo, la prueba estadística de regresión logística indica que no existen diferencias estadísticas significativas entre las dos puntas de parición.

Se ha observado con frecuencia que las mayores tasas de infección ocurren en adultos debido a que en el transcurso del tiempo aumentan las oportunidades de infección en los animales (Dubey et al., 1992; Barberan y Marco, 1997); sin embargo, esto no se pudo verificar en el presente estudio debido probablemente a que sólo se trabajó con hembras de dos años de edad en adelante. Por otro lado, los animales que se encuentran sometidos a inmunosupresión son más proclives a desarrollar enfermedad. Los camélidos sudamericanos presentan inmunosupresión fisiológica durante las épocas de parto y empadre, convirtiéndolos en animales suceptibles a la infección por agentes del medio ambiente (Rojas et al., 1989; Dubey y Lappin, 2000).

Es importante tener presente que los resultados sólo estarían indicando que la infección causada por $T$. gondii en las llamas fue adquirida en algún momento de sus vidas; sin embargo, faltaría profundizar sobre el verdadero rol que cumpliría este parásito en camélidos sudamericanos, en los cuales probablemente podría producir problemas reproductivos y mortalidad en las crías, similares a los que suceden en ovinos y caprinos.

Se concluye que la relativa baja seroprevalencia hallada en el presente estudio se debería a la baja frecuencia de felinos silvestres y domésticos en la zona, y por lo tanto, a una escasa exposición de ooquistes infectivos; así como a las bajas temperaturas y elevada altitud. 


\section{Literatura Citada}

1. Acha, P.; B. Szifres. 1986. Zoonosis y enfermedades transmisibles comunes al hombre y a los animales. $2^{\mathrm{a}}$ ed. $\mathrm{p}$ 645658. Publicación Científica $\mathrm{N}^{\circ}$ 503. OPS.

2. Ameghino, E. 1991. Mortalidad en crías de alpacas. En: Producción de rumiantes menores. p 107. Ed. Martegraf. Lima, Perú.

3. Arthur, G.; E. Noakes; H. Pearson. 1991. Reproducción y obstetricia Veterinaria. 6 ed. p 498-500. Ed. Internamericana McGraw-Hill.

4. Barberan, M.; J.C. Marco. 1997. Patogenia, cuadro clínico y lesional en Toxoplasmosis - Neosporosis. Ovis 52: 35-48.

5. Daniel, W. 1996. Bioestadística base para análisis de las ciencias de la Salud $5^{a}$ ed. p. 205-207, 453-462. Ed. Limusa. México

6. D'angelino, J.L. 1983. Toxoplasmose suína: contribucao para o estudo epidemiológico. Tesis de Doutorado. Fac. Saúde Pública, Univ. Sao Paulo. 112 p.

7. Dubey, J.; L. Rickard; G.L. Zimmerman; D.M. Mulrooney. 1992. Seroprevalence of Toxoplasma gondii in llamas (Lama glama) in the northwest USA. Vet. Parasitol. 44: 295-298.

8. Dubey, J.P.; M. Lappin. 2000. Toxoplasmosis y Neosporosis. En: Enfermedades infecciosas en perros y gatos. 2 ed. p 542-560. McGraw Hill Interamericana. México.

9. Fernández Baca, S. 1991. Avances y perspectivas del conocimiento de los camélidos sudamericanos. FAO. Santiago de Chile. p 327-335.

10. Franco, E. 1998. Manual de crianza de llamas. Pub. Tec. FMV-UNMSM N ${ }^{\circ}$ 33. Lima, Perú.

11. Freij, B.; J. Sever. 1986. Toxoplas-mosis. Red book / Pediatrics in Review / Selfassesment Exercises. February 12(8): 24.

12. Gómez, O. 2002. Determinación de la seroprevalencia de la toxoplasmosis en alpacas y llamas en la Estación Experi- mental del INIA - Puno. Tesis de Médico Veterinario. Facultad de Medicina Veterinaria, Univ. Nacional Mayor de San Marcos. Lima, Perú. 64 p.

13. Góngora, M. 1992. Prevalencia de anticuerpos contra Toxoplasma gondii en las comunidades alpaqueras de Vilcallamas, Bajo Llallagua, Huanacayama y Llusta. Tesis de Médico Veterinario Zootecnista. UNA, Puno. 58 p.

14. Gorman, T.; J. Arancibia; M. Lorca; D. Hird; M. Alcaino. 1999. Seroprevalence of Toxoplasma gondii infección in sheep and alpacas in Chile. Prev. Vet. Med. 40: 143-149.

15. Hartley, J.; C. Marshall, 1967. Toxoplasmosis as a cause of ovine perinatal mortality. N. Z. Vet. J. 5: 119-124.

16. INIA, 1990. Mejoramiento de la producción andina de ovinos y alpacas. Programa de Apoyo a la Investigación Colaborativa en Rumiantes Menores, Perú. INIA. p 185-189.

17. Leguía, G.; H. Samamé; C. Guerrero; M. Rojas; A. Núñez. 1988. Prevalencia de anticuerpos contra Toxoplasma gondii en alpacas. Rev. Camel. Sudamer. IVITA 6: 19-22.

18. Luzón, M.; A. Alonso; A. QuintanillaGonzalo. 1997. Toxoplasmosis Neosporosis. Ovis 52: 11-17.

19. Marcas, C. 2003. Seroprevalencia de Toxoplasma gondii en llamas hembras de la provincia de Melgar-Puno. Tesis de Médico Veterinario. Facultad de Medicina Veterinaria, Univ. Nacional Mayor de San Marcos. Lima. 43 p.

20. Ministerio de Agricultura (MINAG). 1998. Informes estadísticos de producción animal: producción pecuaria e industria avicola. MINAG-OIA. Tomo I. Lima. $16 \mathrm{p}$.

21. Pastor, J.; A. Chávez; E. Casas; E. Serrano. 2003. Seroprevalencia de Toxoplasma gondii en vicuñas de Puno. Rev. Inv. Vet. Perú 14: 79-82.

22. Poma, D. 2003. Seroprevalencia de anticuerpos contra Toxoplasma gondii en alpacas (Lama pacos) de la Unidad 
de Producción de Cochas de la Sais Túpac Amaru. Tesis de Médico Veterinario. Facultad de Medicina Veterinaria, Univ. Nacional Mayor de San Marcos. Lima. $56 \mathrm{p}$.

24. Rojas, M.; I. Lobato; M. Montalvo. 1989. Prevalencia de Toxoplasma gondii en alpacas y llamas. Resúmenes
XII Reunión Científica Anual de la Asociación Peruana de Producción Animal. Lima. $\mathrm{p} 97$.

25. Rojas, M. 1990. Parasitismo de los rumiantes domésticos. Terapia y prevención y modelos para su aprendizaje. $\mathrm{p}$ 326-332. Ed. Mijosa. Lima. 